\title{
On Cauchy-Riemann submanifolds whose local geodesic symmetries preserve the fundamental form
}

\author{
by Sorin Dragomir (Stony Brook, N.Y.) and Mauro CaPursi (Bari)
}

\begin{abstract}
We classify generic Cauchy-Riemann submanifolds (of a Kaehlerian manifold) whose fundamental form is preserved by any local geodesic symmetry.
\end{abstract}

Introduction. Let $\left(M^{2 m}, \bar{g}, J\right)$ be a Hermitian manifold of complex dimension $m$, where $\bar{g}$ denotes the Hermitian metric, while $J$ stands for the complex structure. Let $\Psi: M^{n} \rightarrow M^{2 m}$ be an isometric immersion of a real $n$-dimensional Riemannian manifold $\left(M^{n}, g\right)$ in $M^{2 m}$. Let $E \rightarrow M^{n}$ be the normal bundle of $\Psi$. Then $M^{n}$ is a Cauchy-Riemann (C.R.) submanifold of $M^{2 m}$ if it carries a pair of complementary distributions $\left(D, D^{\perp}\right)$ such that $D$ is holomorphic (i.e. $J_{x}\left(D_{x}\right)=D_{x}, x \in M^{n}$ ) and $D^{\perp}$ is totally real (i.e. $\left.J_{x}\left(D_{x}^{\perp}\right) \subseteq E_{x}, x \in M^{n}\right)$. Let $\tan _{x}$, nor $x$ be the natural projections associated with the direct sum decomposition

$$
T_{x}\left(M^{2 m}\right)=T_{x}\left(M^{n}\right) \oplus E_{x}, \quad x \in M^{n} .
$$

Each C.R. submanifold $M^{n}$ of an (almost) Hermitian manifold $M^{2 m}$ is known to possess a natural $f$-structure $P$ (in the sense of K. Yano [7]) given by $P X=\tan (J X)$. The fundamental 2 -form $\Omega$ of $M^{n}$ is given by

$$
\Omega(X, Y)=g(X, P Y),
$$

for any tangent vector fields $X, Y$ on $M^{n}$. In the present note we are concerned with the following:

Problem. Let $M^{2 m}$ be an (almost) Hermitian manifold. Classify its C.R. submanifolds $M^{n}$ all of whose local geodesic symmetries preserve the fundamental form.

Let $M^{n}$ be such a C.R. submanifold. Set $q=\operatorname{dim}_{\mathbb{R}} D_{x}^{\perp}, x \in M^{n}$. By a result of K. Sekigawa-L. Vanhecke [5], if $M^{n}$ is invariant (i.e. $q=0$ ) then 
$M^{n}$ is a locally symmetric Kaehler manifold. Our contribution regards the generic case (i.e. $q=\operatorname{codim} M^{n}$ ) and consists in the following:

TheOrem. Let $M^{n}$ be an $n$-dimensional generic C.R. submanifold of the Kaehlerian manifold $M^{2 m}$. If all local geodesic symmetries of $M^{n}$ preserve the fundamental form, then $M^{n}$ is locally a Riemannian product $M^{2(n-m)} \times$ $M^{2 m-n}$, where $M^{2(n-m)}$ is a totally geodesic complex submanifold of $M^{2 m}$, while $M^{2 m-n}$ is a totally real submanifold of $M^{2 m}$.

The key ingredient in the proof is a result by A. Gray [4], which provides power series expansions for analytic covariant tensor fields in normal coordinates.

2. Proof of the Theorem. Let $\left(M^{n}, D, D^{\perp}\right)$ be a C.R. submanifold of the Kaehlerian manifold $\left(M^{2 m}, \bar{g}, J\right)$. Let $x \in M^{n}$ and $\left(U_{x}, x^{i}\right)$ a local system of normal coordinates at $x, x^{i}(x)=0,1 \leq i \leq n$. Let $R_{i j k l}$ denote the Riemann-Christoffel tensor field of $\left(M^{n}, g\right)$ and $\nabla_{i}$ covariant differentiation. Let $W_{\alpha_{1} \ldots \alpha_{s}}$ be an analytic covariant tensor field of type $(0, s)$ on $M^{n}$. By a result of A. Gray [4], if $p \in U$ then

$$
\begin{aligned}
& W_{\alpha_{1} \ldots \alpha_{s}}(p)=W_{\alpha_{1} \ldots \alpha_{s}}(x)+\sum_{i=1}^{n}\left(\nabla_{i} W_{\alpha_{1} \ldots \alpha_{s}}\right)(x) x^{i} \\
+ & \frac{1}{2} \sum_{i, j=1}^{n}\left\{\nabla_{i j}^{2} W_{\alpha_{1} \ldots \alpha_{s}}-\frac{1}{3} \sum_{t=1}^{n} \sum_{h=1}^{s} R_{i \alpha_{h} j t} W_{\alpha_{1} \ldots \alpha_{h-1} t \alpha_{h+1} \ldots \alpha_{s}}\right\}(x) x^{i} x^{j} \\
+ & \frac{1}{6} \sum_{i, j, k=1}^{n}\left\{\nabla_{i j k}^{3} W_{\alpha_{1} \ldots \alpha_{s}}-\sum_{t=1}^{n} \sum_{h=1}^{s} R_{i \alpha_{h} j t}\left(\nabla_{k} W_{\alpha_{1} \ldots \alpha_{h-1} t \alpha_{h+1} \ldots \alpha_{s}}\right)\right. \\
- & \left.\frac{1}{2} \sum_{t=1}^{n} \sum_{h=1}^{s}\left(\nabla_{i} R_{j \alpha_{h} k t}\right) W_{\alpha_{1} \ldots \alpha_{h-1} t \alpha_{h+1} \ldots \alpha_{s}}\right\}(x) x^{i} x^{j} x^{k}+\theta\left(x^{4}\right)
\end{aligned}
$$

where $x^{i}=x^{i}(p)$ (cf. also B. Y. Chen-L. Vanhecke [1], p. 31).

Let $\gamma: r \rightarrow \exp _{x}(r X) \in U_{x}$ be a geodesic (parametrized by arc length) of $\left(M^{n}, g\right)$, where $X \in T_{x}\left(M^{n}\right),\|X\|=1$. Let $\Omega$ be the fundamental 2 -form of $M^{n}$. We work under the basic assumption that each local geodesic symmetry $f: \exp _{x}(r X) \rightarrow \exp _{x}(-r X)$ preserves $\Omega$, i.e. $f^{*} \Omega=\Omega$, or

$$
\Omega_{i j}\left(\exp _{x}(r X)\right)=\Omega_{i j}\left(\exp _{x}(-r X)\right) .
$$

The local parametric equations (in normal coordinates) of $\gamma$ are $x^{i}(r)=$ $r X^{i}, 1 \leq i \leq n$. Thus the power series expansion formula (2.1) leads 
to the expansion

$$
\begin{aligned}
& \Omega_{\alpha_{1} \alpha_{2}}(\gamma(r))=\Omega_{\alpha_{1} \alpha_{2}}(x)+\sum_{i=1}^{n} X^{i}\left(\nabla_{i} \Omega_{\alpha_{1} \alpha_{2}}\right)(x) r \\
& +\frac{1}{2} \sum_{i, j=1}^{n} X^{i} X^{j}\left\{\nabla_{i j}^{2} \Omega_{\alpha_{1} \alpha_{2}}-\frac{1}{3} \sum_{t=1}^{n}\left(R_{i \alpha_{1} j t} \Omega_{t \alpha_{2}}+R_{i \alpha_{2} j t} \Omega_{\alpha_{1} t}\right)\right\}(x) r^{2} \\
& +\frac{1}{6} \sum_{i, j=1}^{n} X^{i} X^{j} X^{k}\left\{\nabla_{i j k}^{3} \Omega_{\alpha_{1} \alpha_{2}}-\sum_{t=1}^{n}\left(R_{i \alpha_{1} j t} \nabla_{k} \Omega_{t \alpha_{2}}+R_{i \alpha_{2} j t} \nabla_{k} \Omega_{\alpha_{1} t}\right)\right. \\
& \left.-\frac{1}{2} \sum_{t=1}^{n}\left(\Omega_{t \alpha_{2}} \nabla_{i} R_{j \alpha_{1} k t}+\Omega_{\alpha_{1} t} \nabla_{i} R_{j \alpha_{2} k t}\right)\right\}(x) r^{3}+\theta\left(r^{4}\right) .
\end{aligned}
$$

Let $\left\{e_{i}\right\}_{1 \leq i \leq n}$ be an orthonormal basis of $T_{x}\left(M^{n}\right)$ such that $e_{1}=X$. We suppose the normal coordinates at $x$ have been chosen such that $\partial /\left.\partial x^{i}\right|_{x}=$ $e_{i}, 1 \leq i \leq n$. By straightforward computation our (2.3) turns into

$$
\begin{aligned}
\Omega_{i j}(\gamma(r))= & \left\langle e_{i}, P_{x} e_{j}\right\rangle+\left\langle e_{i},(\nabla P)_{x}\left(X, e_{j}\right)\right\rangle r \\
& +\frac{1}{2}\left\langle e_{i},\left(\nabla^{2} P\right)_{x}\left(X, X, e_{j}\right)-\frac{1}{3}(R P+P R)_{x} e_{j}\right\rangle r^{2} \\
& +\frac{1}{6}\left\langle e_{i},\left(\nabla^{3} P\right)_{x}\left(X, X, X, e_{j}\right)-R_{x}(\nabla P)_{x}\left(X, e_{j}\right)\right. \\
& -(\nabla P)_{x}\left(X, R_{x} e_{j}\right)-\frac{1}{2}\left\{\left(\nabla_{X} R\right)\left(P_{x} e_{j}, X\right) X\right. \\
& \left.\left.+P_{x}\left(\nabla_{X} R\right)\left(e_{j}, X\right) X\right\}\right\rangle r^{3}+\theta\left(r^{4}\right)
\end{aligned}
$$

where $\langle\rangle=,g_{x}$ and $R_{x}$ denotes the transformation $R_{x} v=R(v, X) X$, $v \in T_{x}\left(M^{n}\right)$. Next (2.2) and (2.4) furnish $\nabla P=0$, i.e. the canonical $f$-structure of $M^{n}$ is parallel and

$$
\left(\nabla_{X} R\right)(P Y, X) X+P\left(\nabla_{X} R\right)(Y, X) X=0
$$

for any $Y \in T_{x}\left(M^{n}\right)$. Set $F Z=\operatorname{nor}(J Z)$ for any tangent vector field $Z$ on $M^{n}$. Then $F$ is a normal bundle valued 1 -form on $M^{n}$ vanishing on the holomorphic distribution. Set $t \xi=\tan (J \xi), f \xi=\operatorname{nor}(J \xi)$, for any cross-section $\xi$ in $E \rightarrow M^{n}$. Clearly, if $M^{n}$ is generic $(q=2 m-n)$ then $f=0$. Let $\sigma$ be the second fundamental form of $\Psi$ and $a_{\xi}$ the Weingarten operator (associated with the normal section $\xi$ ). Let $\bar{\nabla}$ be the Levi-Civita connection of $\left(M^{2 m}, \bar{g}\right)$. Yet $\bar{g}$ is Kaehlerian, i.e. $\bar{\nabla} J=0$; by the Gauss and Weingarten formulae (see e.g. eqs. (1.1)-(1.2) of [9], p. 19) and identification of tangential, respectively normal, components, one obtains

$$
\begin{aligned}
& \left(\nabla_{X} P\right) Y=a_{F Y} X+t \sigma(X, Y), \\
& \left(\nabla_{X} F\right) Y=-\sigma(X, P Y)
\end{aligned}
$$

for any tangent vector fields $X, Y$ on $M^{n}$. As $P$ is parallel, $F P=0$ and 
$t=J$, formula $(2.6)$ gives

$$
\sigma(X, P Y)=0
$$

and by (2.7), $F$ is parallel, too. As a consequence both the holomorphic and totally real distributions are parallel and thus $M^{n}$ is locally a Riemannian product. It is easily seen that (2.8) also yields that $M^{2(n-m)}$ is totally geodesic.

Remarks. (i) Let $M^{2 m}$ be a complete simply connected complex space-form (of constant holomorphic curvature $c$ ). Combining our Theorem with a result by K. Yano-M. Kon [8], one shows that if $M^{n}$ is a complete generic submanifold obeying (2.2) then either $M^{n}$ is an m-dimensional totally real (i.e. $P=0$ ) submanifold of $M^{2 m}$, or $c=0$ and $M^{n}$ is congruent to $\mathbb{C}^{n-m} \times M^{2 m-n}$, where $M^{2 m-n}$ is a totally real submanifold of $\mathbb{C}^{m}$.

(ii) The curvature identity (2.5) does not contribute further to the classification of generic submanifolds (subject to (2.2)) of complex space-forms. Indeed, by remark (i), either $P=0$ and thus (2.5) is identically satisfied, or $c=0$ and then (by the Gauss eq. (1.10) of [9], p. 78), $R(Y, Z) W=$ $a_{\sigma(Z, W)} Y-a_{\sigma(Y, W)} Z$ for any tangent vector fields $Y, Z, W$ on $M^{n}$. As $M^{n}$ is generic, any normal field $\xi$ may be written as $\xi=F Y$ for some $Y$ tangent to $M^{n}$. Thus (2.6) and (2.8) yield $a_{\xi} P Y=0$ for any $Y$, $\xi$. Consequently, $R(P Y, Z) W=R(Y, P Z) W=R(Y, Z) P W=0$ and (2.5) turns into $P \nabla_{X}\left(R(Y, X) i^{\perp} X\right)=0$, where $i^{\perp}=-t F$; now this is identically satisfied, as $D^{\perp}$ is parallel.

(iii) Let $M^{2 m}$ be a locally conformal Kaehler manifold (see e.g. I. Vaisman [6]). Let $M^{n}$ be a generic C.R. submanifold subject to (2.2). Repeating the arguments in the proof of our Theorem, we obtain $\nabla P=0$. Then, by a result of [2; II, Th. 1, p. 2], if the 1-form $\omega$ induced by the Lee form of $M^{2 m}$ has no singular points (i.e. $\omega_{x} \neq 0$, for any $x \in M^{n}$ ) then $M^{n}$ is a totally real submanifold of $M^{2 m}$ (see also [3]).

\section{References}

[1] B. Y. Chen and L. Vanhecke, Differential geometry of geodesic spheres, J. Reine Agnew. Math. 325 (1981), 28-67.

[2] S. Dragomir, Cauchy-Riemann submanifolds of locally conformal Kaehler manifolds, I, II, Geom. Dedicata 28 (1988), 181-197; Atti Sem. Mat. Fis. Univ. Modena 37 (1989), 1-11.

[3] —, On submanifolds of Hopf manifolds, Israel J. Math. (2) 61 (1988), 199-210.

[4] A. Gray, The volume of a small geodesic ball in a Riemannian manifold, Michigan Math. J. 20 (1973), 329-344.

[5] K. Sekigaw and L. Vanhecke, Symplectic geodesic symmetries on Kaehler manifolds, Quart. J. Math. Oxford Ser. (2) 37 (1986), 95-103. 
[6] I. Vaisman, Locally conformal Kähler manifolds with parallel Lee form, Rend. Mat. 12 (1979), 263-284.

[7] K. Yano, On a structure defined by a tensor field of type $(1,1)$ satisfying $f^{3}+f=0$, Tensor (N.S.) 14 (1963), 99-109.

[8] K. Yano and M. Kon, Generic submanifolds, Ann. Mat. Pura Appl. 123 (1980), 59-92.

[9] - - C CR Submanifolds of Kaehlerian and Sasakian Manifolds, Progr. Math. 30, Birkhäuser, Boston 1983.

DEPARTMENT OF MATHEMATICS

STATE UNIVERSITY OF NEW YORK

AT STONY BROOK

STONY BROOK, NEW YORK 11794-3651

U.S.A.
DIPARTIMENTO DI MATEMATICA UNIVERSITÀ DEGLI STUDI DI BARI 70125 BARI, ITALY

Reçu par la Rédaction le 15.1.1990 